\title{
Atoms interacting with intense, high-frequency laser pulses: Effect of the magnetic-field component on atomic stabilization
}

\author{
J. R. Vázquez de Aldana, ${ }^{1}$ N. J. Kylstra, ${ }^{2}$ L. Roso, ${ }^{1}$ P. L. Knight, ${ }^{3}$ A. Patel, ${ }^{3}$ and R. A. Worthington ${ }^{2}$ \\ ${ }^{1}$ Departamento de Física Aplicada, Universidad de Salamanca, E-37008 Salamanca, Spain \\ ${ }^{2}$ Department of Physics, Science Laboratories, University of Durham, Durham DH1 3LE, United Kingdom \\ ${ }^{3}$ Optics Section, Blackett Laboratory, Imperial College, London SW7 2BW, United Kingdom
}

(Received 20 December 2000; published 7 June 2001)

\begin{abstract}
At sufficiently high laser intensities, the magnetic-field component of the laser field can strongly influence the stabilization of atoms in the high-frequency regime by inducing motion along the laser pulse propagation direction. Using a two-dimensional model atom, we investigate how the duration of the laser pulse affects stabilization in this nondipole regime. Results obtained using a fully spatially dependent vector potential are compared to a long-wavelength approximation. We also discuss nondipole effects when the atom interacts with two counterpropagating pulses.
\end{abstract}

DOI: 10.1103/PhysRevA.64.013411

PACS number(s): 42.50.Hz, 32.80.Rm, 32.80.Fb

\section{INTRODUCTION}

The surprising stability against ionization of atoms interacting with an intense high-frequency laser field has been the focus of much research over the last decade [1]. Termed atomic stabilization, many aspects of this phenomenon can be understood by performing a Kramers-Henneberger (KH) transformation to the rest frame of a classical electron in the laser field. In particular, by developing a high-frequency Floquet theory (HFFT) in the KH frame [2-4], stabilization can be seen to have its origin in the rapid quiver motion of the laser-driven electron about the nucleus. This allows the electron dynamics to be described by an effective cycle-averaged potential. Subsequent $a b$ initio Floquet calculations confirmed that ionization rates decrease with increasing intensity in a high-frequency field [5]. By directly integrating the time-dependent Schrödinger equation numerically, simulations in one [6], two [7], and three dimensions (with cylindrical symmetry) [8] demonstrated reductions in the ionization probability with increasing laser intensity when an atom interacts with realistic laser pulses having a finite duration [9]. Further work has been carried out in order to elucidate the effects of the pulse shape and duration [10-12]. Investigations of stabilization have stimulated the development of $a b$ initio numerical methods to simulate atoms driven by intense laser pulses. However experimentally, the situation has been hampered by the lack of ultraintense far-UV laser sources. Only evidence of atomic stabilization of Rydberg states has been observed so far [13].

Until recently, most simulations of stabilization in the high-frequency, high-intensity regime have been performed using the electric dipole approximation. In this approximation, the spatial dependence of the vector potential is neglected, so that the magnetic-field component of the laser pulse vanishes and an electron in the laser field undergoes simple harmonic motion along the laser polarization direction. However, as the laser intensity increases, the dipole approximation breaks down. Magnetic-field and relativistic effects that can alter the stabilization dynamics become im- portant [14]. This has been investigated in atomic hydrogen, where calculations beyond the dipole approximation have been carried out [15]. However, at the frequencies and intensities investigated, nondipole effects were found to be small. Classical Monte Carlo simulations are less intensive computationally, and therefore allow the influence of magnetic-field and relativistic effects to be studied over a wide range of laser parameters. In this way, it was shown that these effects can indeed be detrimental to stabilization [16]. In addition, relativistic and magnetic-field effects have been studied using a two-dimensional Dirac model [17]. The difficulty of nondipole, nonrelativistic calculations for atomic hydrogen, and numerically solving the Dirac equation in two dimensions, has led to recent interest in using a nonrelativistic two-dimensional model to investigate stabilization [18-21]. The effects of the magnetic-field component of the laser are accounted for in this model, and its reduced dimensionality means that laser-atom interactions beyond the dipole approximation can be readily carried out [18]. However, being nonrelativistic, the model describes electron dynamics only to order $1 / c$. Nevertheless, nondipole effects can be significant enough to modify the stabilization of an atom in the nonrelativistic regime [18-22].

The HFFT indicates that stabilization is to be expected when the classical excursion of the electron is large compared to the size of the unperturbed atom, and the photon energy is sufficiently greater than the binding energy of the atom in the laser field [4]. This adiabatic theory, very elegantly formulated using the $\mathrm{KH}$ frame transformation, shows that the atomic structure of the atom in an intense, high-frequency laser field is essentially determined by the time average of an effective oscillating Coulomb potential in the KH frame [4]. This static potential is referred to as the $\mathrm{KH}$ potential. As the classical excursion amplitude increases, the binding energy of the ground state of the $\mathrm{KH}$ potential decreases. At the same time, the wave function becomes spatially extended, and its ionization rate decreases. However, if the excursion becomes too large, the electron velocity in the electric-field polarization direction can couple-due to the Lorentz force-with the magnetic-field component of the la- 
ser, thereby modifying the electron's trajectory. This motion has been considered within the framework of the HFFT [23], and it was shown that very large excursion amplitudes are required to substantially modify the atomic structure in the laser field. For short intense laser pulses, the evolution of the atom in the field is in general not adiabatic. In this case, the magnetic-field can introduce a non-negligible force along the propagation direction that can displace the electronic trajectory many atomic units in the propagation direction $[18,20]$. This is detrimental to stabilization, as we discuss in the present paper.

We first introduce the two-dimensional model atom on which our study is based. We next discuss the form of the vector potential used to describe the laser pulse, and develop a long-wavelength approximation in the stabilization regime. Results obtained by numerically integrating the timedependent Schrödinger equation are then presented. Since the effect of the magnetic drift on the atomic system depends on how quickly the laser field is turned on and off, magneticfield effects over a range of laser pulse durations are investigated. We also numerically test the accuracy of the longwavelength approximation for a single laser pulse and for two counterpropagating pulses. In both these cases, the relatively simple form of the long-wavelength Hamiltonian allows some general conclusions to be drawn concerning how the influence of the magnetic-field component of the laser depends on the laser pulse duration in the stabilization regime.

\section{TWO-DIMENSIONAL MODEL ATOM}

We assume that the dynamics of the system are nonrelativistic, and therefore can be described by the timedependent Schrödinger equation

$$
i \frac{\partial}{\partial t} \Psi(\mathbf{r}, t)=\left(\frac{1}{2}[\mathbf{p}+\mathbf{A}(\eta)]^{2}+V(r)\right) \Psi(\mathbf{r}, t),
$$

with $\mathbf{r}=(x, y)$ and $\eta=\omega(t-x / c)$, where $\omega$ is the angular frequency of the laser field and $c$ is the speed of light. The laser pulse is taken to be linearly polarized along $\hat{\mathbf{y}}$ so that the laser pulse propagates in the $\hat{\mathbf{x}}$ direction. The magneticfield component of the laser is therefore polarized along $\hat{\mathbf{z}}$. The vector potential $\mathbf{A}(\eta)$ describes the laser pulse, and is discussed in more detail in Sec. III. The scalar potential is zero in the gauge used here. We point out that the motion of a free electron, initially at rest, interacting with a laser pulse described by the vector potential $\mathbf{A}(\eta)$, is confined to the $x-y$ plane. The spin of the electron is neglected, since it is not expected to play an important role in governing the dynamics for the laser parameters considered here [24]. Unless otherwise indicated, we use atomic units (a.u.).

To model the Coulomb interaction between the electron and the nucleus, we employ a two-dimensional "soft-core" potential [7]

$$
V(r)=-\frac{1}{\sqrt{r^{2}+a^{2}}} .
$$

The smoothing parameter $a$ is used to avoid problems in two dimensions associated with the Coulomb singularity at the origin. We have set $a=0.80$, so that the ground state has the same binding energy as atomic hydrogen, i.e., -0.50 . The potential supports an infinite number of Rydberg series of bound states. Starting from the ground state of the system, Eq. (1) is numerically integrated on an uniform grid using either a split-step operator method or a Crank-Nicholson scheme. Both methods are widely used for studying intense laser-atom interactions, and they can be easily modified to account for the spatially dependent vector potential.

The probability that the atom does not ionize during the laser pulse is determined by projecting the wave function at the end of the laser pulse onto the bound field-free states of the atom,

$$
P_{\text {survival }}=\sum_{n, m}\left|\left\langle\psi_{n m} \mid \Psi(\tau)\right\rangle\right|^{2}
$$

where $\tau$ is the pulse duration. The field-free states $\left|\psi_{n m}\right\rangle$ are labeled by their principal quantum number $n$ and azimuthal quantum number $m$. In cylindrical coordinates $\psi_{n m}(\mathbf{r})$ $=(2 \pi)^{1 / 2} R_{n m}(r) \exp (-i m \phi)$, so that the radial functions are solutions of the differential equation

$$
\left[-\frac{1}{2 r} \frac{\partial}{\partial r}\left(r \frac{\partial}{\partial r}\right)+\frac{m^{2}}{2 r^{2}}+V(r)-E_{n m}\right] R_{n m}(r)=0 .
$$

This equation was solved by expanding the $R_{n m}(r)$ in terms of $B$-spline functions, and then diagonalizing the resulting $B$-spline representation of the radial Hamiltonian.

For our calculations using the Crank-Nicholson method, the grid in both $x$ and $y$ was comprised of 2000 points, with a grid spacing of 0.2. A mask function was employed in the 200 grid points along the edge of the grid. For the splitoperator calculations, 1024 points and a grid spacing of 0.44 were used in $x$ and $y$. A mask was applied on the first and last 225 grid points along $x$, and the first and last 325 grid points along $y$. The survival probability and the populations in the bound states at the end of the pulse were calculated by expanding the radial functions, $R_{n m}(r)$, in terms of 425 $B$-spline functions for each $m$, with $m=-7, \ldots, 7$. For $m$ $=0,12$ of the 425 radial functions corresponded to bound states, while for $|m|=7$ seven were bound states. The lowlying states obtained using a $B$-spline expansion correspond to the physical states of the system, and the highly excited states are "pseudostates" that can account for the remaining excited states in the Rydberg series, as can be verified by evaluating sum rules [25]. Survival probabilities calculated from wave functions obtained using the Crank-Nicholson and split-operator methods typically agree to better than $5 \%$.

\section{LASER FIELD}

The laser field is described classically using a fully spatially dependent vector potential that includes retardation, 


$$
\mathbf{A}(\eta)=-\frac{1}{\omega} \int_{-\infty}^{\eta} \mathbf{E}\left(\eta^{\prime}\right) d \eta^{\prime}
$$

with

$$
\mathbf{E}(\eta)=E_{0} f(\eta) \sin \eta \hat{\mathbf{y}}
$$

The function $f(\eta)$ is the laser pulse envelope, which is taken to be trapezoidal. This function is nonzero for $0<\eta<\omega \tau$. The maximum electric-field strength of the pulse is $E_{0}$. In order to investigate the effects of the pulse duration $\tau$, we have performed simulations for a range of durations while keeping the same form of the pulse envelope. In particular, we have used a pulse with an $N$-cycle linear turn-on, $2 N$ cycles of constant intensity, and a $N$-cycle turn-off, with $N$ $=1-10$. This $N+2 N+N$ trapezoidal pulse has a total duration of $\tau=4 N(2 \pi) / \omega$. Results obtained using the traveling wave described by Eq. (5) will be referred to throughout the paper as the nondipole results.

In addition to using the spatially dependent vector potential, thereby completely accounting for the magnetic-field component and retardation, we have integrated the timedependent Schrödinger equation using the dipole approximation, i.e., setting $\mathbf{A}(\eta)=\mathbf{A}(\omega t)$, and taking $\mathbf{A}(\omega t)$ to defined over all space. We have also considered the case of two linearly polarized, counterpropagating pulses. In this case the electric field of the two pulses is given by with $\eta^{\prime}=\omega(t+x / c)$. At the origin, where the nucleus is located, the electric field oscillates with the same amplitude as for the single pulse case, while the magnetic-field vanishes: the antinode of the electric field is precisely at the node of the magnetic-field. Finally, we have performed calculations using a long-wavelength approximation discussed in Sec. IV.

\section{LONG-WAVELENGTH APPROXIMATION}

Because of its nonrelativistic origin, the Schrödinger equation is invariant under Galilean transformations. On the other hand, the laser fields considered in the nondipole case [see Eq. 6] are Lorentz invariant, as they describe a laser pulse propagating with speed $c$ that satisfies the Maxwell equations. This may lead to inconsistencies in our model [26]. The first relativistic correction to the kinetic-energy operator is of order $1 / c^{2}$, while the Schrödinger equation is accurate only up to first order in $1 / c$. However, the different transformation properties of the laser pulse and the Schrödinger wave function will not give rise to problems as long as we remain in an intensity and frequency regime where the dynamics of the system remains nonrelativistic.

Indeed, it has been shown that a nonrelativistic regime exists where nondipole effects can become important $[18,20]$. Using the Lorentz force equations for a free electron that is initially at rest and interacting with a laser pulse, the force on and velocity of the electron along the propagation axis are, to lowest order in $1 / c$,

$$
\mathbf{E}\left(\eta, \eta^{\prime}\right)=\frac{E_{0}}{2}\left[f\left(\eta^{\prime}\right) \sin \eta^{\prime}+f(\eta) \sin \eta\right] \hat{\mathbf{y}}
$$
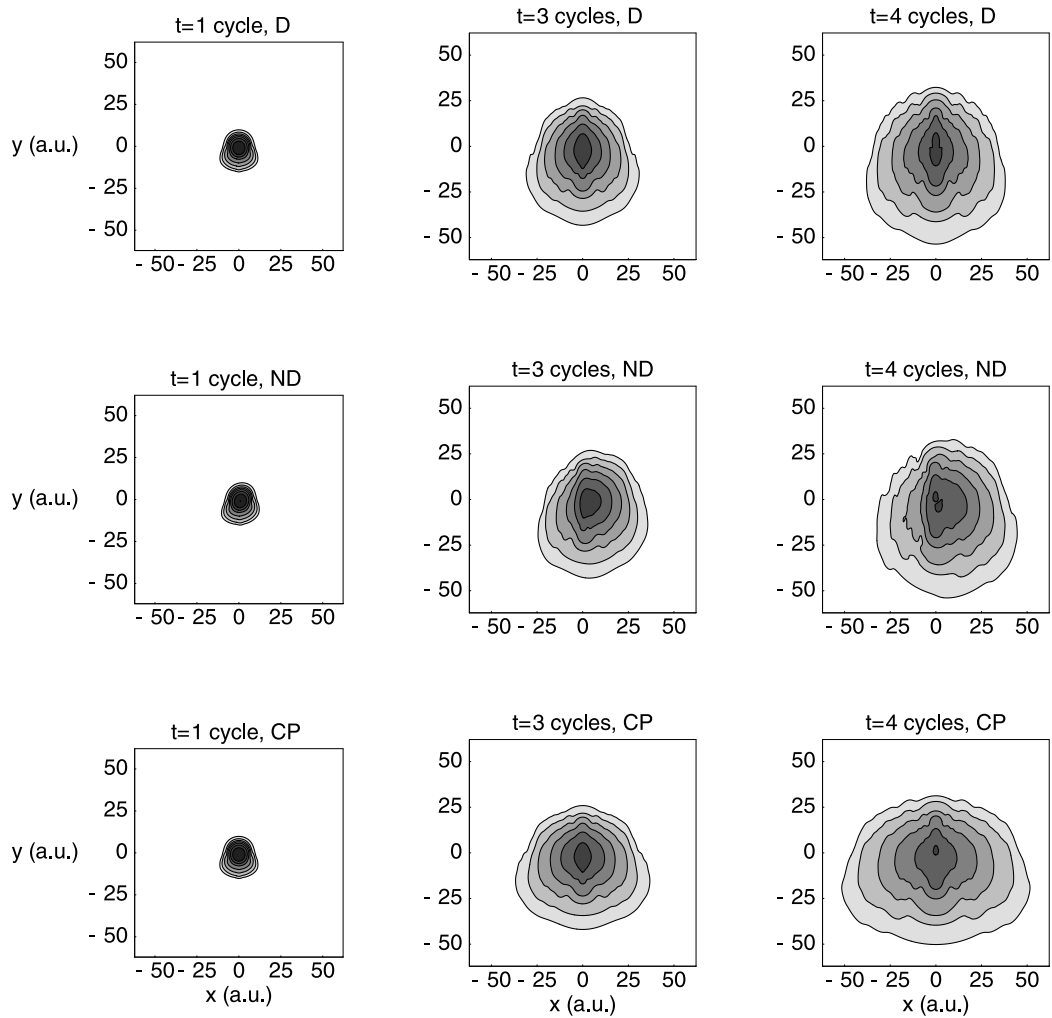

$$
F_{x}(t)=\frac{1}{2 c} \frac{d}{d t} A^{2}(\omega t)
$$

FIG. 1. Contour plots of the probability density during a four-cycle trapezoidal pulse (one cycle turn-on, two cycles constant intensity, and one cycle turn-off), with $E_{0}=15$ (corresponding to an intensity of $7.9 \times 10^{18} \mathrm{~W} \mathrm{~cm}^{-2}$ ) and $\omega=1$ $(\hbar \omega=27.2 \mathrm{eV})$. The electric field of the laser field is polarized along the $y$ direction, and the pulse propagates along the positive $x$ direction. The upper row contains results obtained in the dipole approximation (D), while the middle row and low rows display the nondipole (ND) and counterpropagating (CP) results, respectively. The times of the snapshots are after the end of the turn-on (left column), at the beginning of the turn-off (middle column), and at the end of the pulse (right column). The contour lines are on a logarithmic scale. The same scale is used for all the plots, with the darker regions indicating high probability densities. 

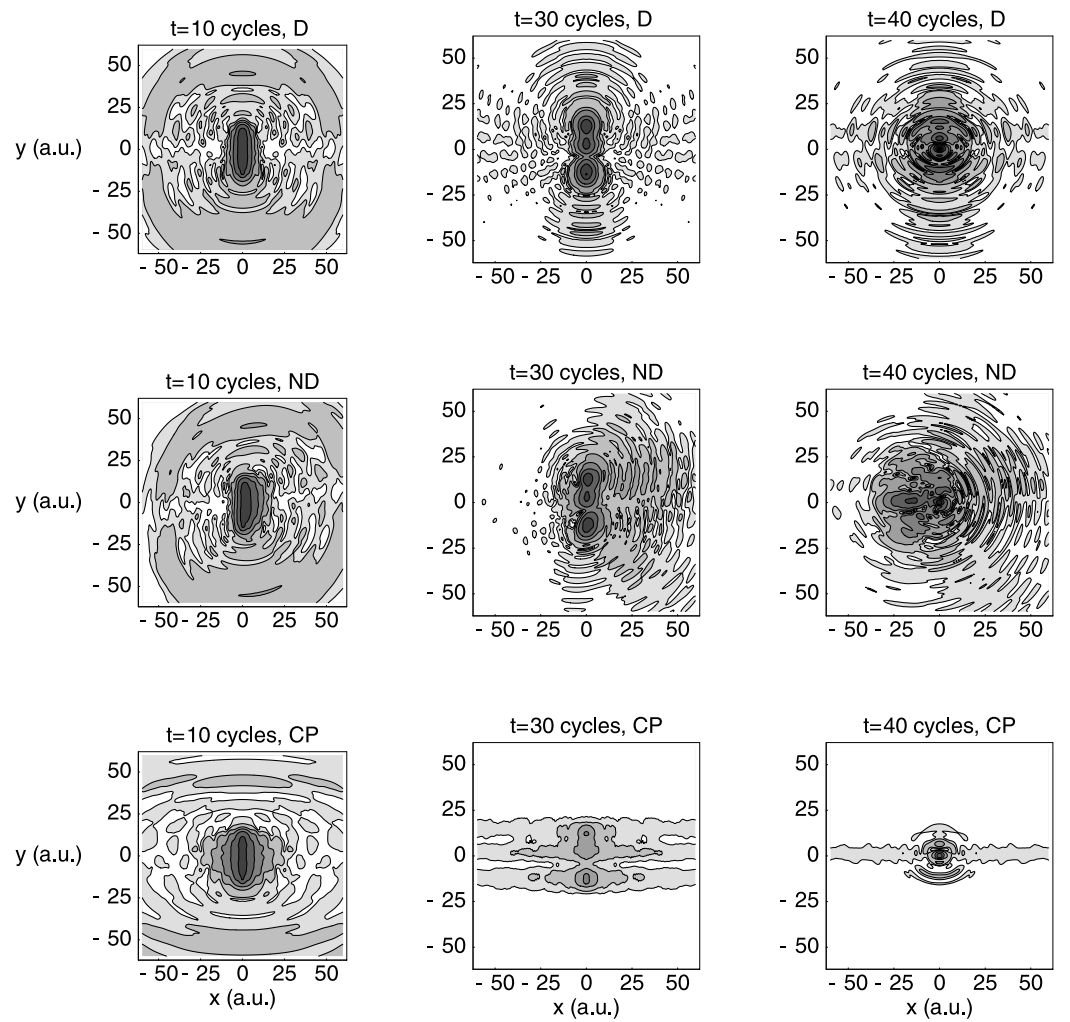

$$
v_{x}(t)=\frac{1}{2 c} A^{2}(\omega t)
$$

In this approximation, both the electric and magnetic fields of the laser are spatially homogeneous. Note that the magnetic-field induces a velocity in the propagation direction that is never negative. The displacement in the propagation direction per laser cycle is, correct to order $1 / c$,

$$
\chi_{0} \approx E_{0}^{2} /\left(c \omega^{3}\right) .
$$

This result is valid when the ratio of the ponderomotive energy of the electron to its rest mass, $E_{0}^{2} /\left(4 \omega^{2} c^{2}\right)$, is small, i.e., the electron dynamics is essentially nonrelativistic. The displacement of the electron in the propagation direction will be important when it becomes comparable to the width of the electron wave packet. Calling $\beta$ the ratio of the maximum velocity of the electron in the laser field to the speed of light, $\beta=E_{0} / \omega c$, these two criteria imply that we must have

$$
\frac{1}{4} \beta^{2} \ll 1<\frac{1}{k a} \beta^{2}
$$

where $a$ is the width of the wave packet along the propagation direction, and $k=\omega / c$. This, in turn, implies that $k a$ $\ll 1$. One recognizes this as the usual requirement for the applicability of the electric dipole approximation. However, in contrast to the dipole approximation, the magnetic-field component of the pulse is now no longer zero.

A long-wavelength approximation can also be obtained by expanding the vector potential in powers of $1 / c$, and retaining only the lowest-order contribution beyond the dipole
FIG. 2. Same as Fig. 1, but for a 40-cycle trapezoidal pulse (ten-cycle turn-on, 20-cycle constant intensity, and ten-cycle turn-off). The times of the snapshots are again at the end of the turn-on (left column), at the beginning of the turn-off (middle column), and at end of the pulse (right column). The broad, light gray areas of low density seen after ten cycles are due to photoionization during the relatively long pulse turn-on. Striking differences are observed between the dipole (D), nondipole (ND), and counterpropagating $(\mathrm{CP})$ results. approximation in Hamiltonian. For a pulse of duration $\tau$, the vector potential is expanded as

$$
\mathbf{A}(\eta)=\left\{\begin{array}{cc}
\mathbf{A}(\omega t)+\left(\frac{x}{c}-\frac{x^{2}}{2 c^{2}} \frac{d}{d t}+\cdots\right) \mathbf{E}(\omega t), & 0<\eta<\omega \tau \\
0 & \text { otherwise. }
\end{array}\right.
$$

The wavelength $\lambda=2 \pi c / \omega$ is much larger than the relevant atomic dimensions along the propagation direction; therefore, we can neglect retardation, and simply assume that

$$
\mathbf{A}(\eta)=\mathbf{A}(\omega t)+\left(\frac{x}{c}-\frac{x^{2}}{2 c^{2}} \frac{d}{d t}+\cdots\right) \mathbf{E}(\omega t)
$$

for all values of $\eta$. As the motion of a classical electron in the laser pulse can be well approximated by a solution of the Lorentz equations that is correct to order $1 / c$, we consider the following expression, correct to order $1 / c$, for the Hamiltonian of the system:

$$
H \simeq \frac{1}{2}[\mathbf{p}+\mathbf{A}(\omega t)]^{2}+\frac{x}{c}[\mathbf{p}+\mathbf{A}(\omega t)] \cdot \mathbf{E}(\omega t)+V(r) .
$$

This expression can be further simplified by making use of the fact that the momentum distribution of the electron wave packet in the high-frequency, high-intensity regime is strongly centered about zero, particularly along the laser polarization axis $y$. Therefore, we can make the additional approximation 


$$
H \simeq \frac{1}{2}[\mathbf{p}+\mathbf{A}(\omega t)]^{2}+\frac{x}{c} \mathbf{A}(\omega t) \cdot \mathbf{E}(\omega t)+V(r) .
$$

Calculations using this Hamiltonian have been carried out for a two-dimensional model atom $[19,21]$ and for atomic hydrogen [27]. A similar approximation to order $1 / c$ in the length gauge that neglects retardation effects was considered in Ref. [28].

For the counterpropagating pulses, the terms in $1 / c$ cancel at the points in space where the electric fields of the two pulses are exactly the same. However, by ignoring the finite spatial extend of the pulses (i.e., ignoring retardation), the terms proportional to $1 / c$ cancel everywhere, and the longwavelength approximation for the Hamiltonian describing the atom interacting with two counterpropagating pulses is

$$
H \simeq \frac{1}{2}[\mathbf{p}+\mathbf{A}(\omega t)]^{2}-\frac{1}{2} \frac{x^{2}}{c^{2}}[\mathbf{p}+\mathbf{A}(\omega t)] \cdot \frac{d}{d t} \mathbf{E}(\omega t)+V(r) .
$$

As before, this can be further simplified to

$$
H \simeq \frac{1}{2}[\mathbf{p}+\mathbf{A}(\omega t)]^{2}-\frac{1}{2} \frac{x^{2}}{c^{2}} \mathbf{A}(\omega t) \cdot \frac{d}{d t} \mathbf{E}(\omega t)+V(r) .
$$

Numerical results obtained using the approximate longwavelength Hamiltonians given by Eqs. (14)-(17) will be discussed in Secs. VI and VII below.

\section{NUMERICAL RESULTS}

All of the numerical results presented here were obtained for an angular frequency of $\omega=1(\hbar \omega=27.2 \mathrm{eV})$. This is a typical value used in studies of atomic stabilization, and can be considered a compromise between the asymptotically high frequencies required to give nondecaying bound states in the field, and what can be expected in the near future from intense free-electron laser sources.

Figures 1 and 2 show contour plots of the calculated probability density $|\Psi(\mathbf{r}, t)|^{2}$ in the $x-y$ plane at different times during the pulse. The maximum electric-field amplitude was taken to be $E_{0}=15$, corresponding to a peak intensity of $7.9 \times 10^{18} \mathrm{~W} \mathrm{~cm}^{-2}$. This intensity is sufficiently high to give rise to a non-negligible displacement in the propagation direction, while it is low enough so that the quiver motion of the electron remains nonrelativistic [see Eq. (11)]. The same logarithmic scale is used in all of the plots. The pulse propagates in the positive $x$ direction, and the electric field of the laser is polarized along the vertical axis. The left, center, and right columns, respectively, are the probability densities at the end of the turn-on, the beginning of the turn-off, and at the end of the pulse. The upper row corresponds to the results obtained in the dipole (D) approximation, the middle row to the nondipole (ND) case (i.e., the traveling-wave case with the magnetic field component accounted for), and the lower row to the counterpropagating $(\mathrm{CP})$ case.

In Fig. 1, results for the shortest pulse considered, a fourcycle $(1+2+1$ cycles $)$ pulse, are shown. The three plots in the left-hand column (the probability density at the end of the turn-on) are similar, with the nondipole wave packet already showing a small left-right asymmetry. However, by the end of the pulse, the effect of the magnetic-field drift in the forward direction for the nondipole case, and in the forward and backward directions for the counter-propagating case, is evident. As the peak intensity is rapidly reached for this short pulse, the electron behaves nearly as a free, spreading wave packet in the field.

Results for a 40-cycle $(10+20+10$ cycles $)$ pulse, the longest pulse considered, are shown in Fig. 2. The remaining pulse parameters are the same as in Fig. 1. The columns correspond to the wave-function density after ten cycles (end of the turn-on), 30 cycles (beginning of the turn-off), and 40 cycles (end of the pulse), respectively. The same logarithmic scale is used as in Fig. 1. The influence of the magneticfielddrift in the nondipole case is now more striking, with the electron probability distribution being pulled out in the pulse propagation direction. For counterpropagating pulses, the electron readily ionizes in the positive and negative $x$ directions after the pulse turn-on. Counterpropagating pulses do not improve the stability of the atom, as will be discussed further below.

Surface plots of the probability density for a 16-cycle (4
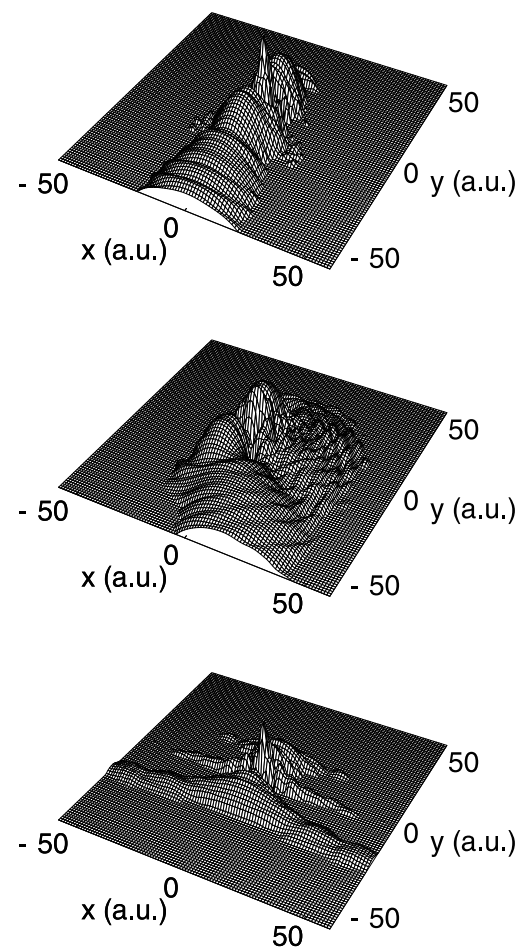

FIG. 3. Surface plots of the probability density after 12 cycles of a 16-cycle trapezoidal pulse. The remaining laser parameters are the same as in Fig. 1. For this pulse duration, the differences between the dipole and nondipole simulations are the largest. In the dipole approximation (upper plot), the wave function remains largely localized along the electric-field polarization axis. This is no longer true when the magnetic-field component is accounted for (nondipole results, middle plot). For the counterpropagating pulses (lower plot), a significant fraction of the population has left the vicinity of the nucleus due to the antinode instability there (see the text). 

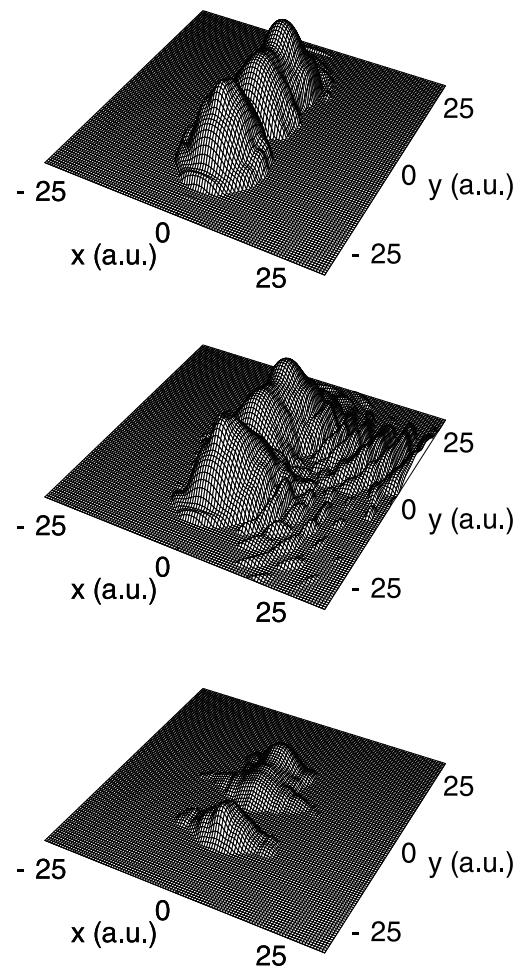

FIG. 4. Same as Fig. 3, except that the probability density is shown after 30 cycles of a 40-cycle trapezoidal pulse. For this longer pulse, the wave packet calculated using the dipole approximation (upper plot) remains localized near the nucleus between $-\alpha_{0}$ and $+\alpha_{0}$ along the electric-field polarization axis, where $\alpha_{0}$ $=15$ is the classical electron excursion amplitude. When the influence of the magnetic field is included (nondipole results, middle plot), this is no longer true. The magnetic-field-induced drift leads to the ejection of a series of ionizing wave packets along the laser propagation direction. For the counterpropagating pulse (lower plot), most of the population has left the vicinity of the nucleus by tunneling (see text).

$+8+4$ cycles) pulse at the beginning of the turn-off (after 12 cycles) are shown in Fig. 3. The upper, middle, and lower plots correspond, as before, to the dipole, nondipole, and counterpropagating cases. The vertical scale is again logarithmic. This alternative way of viewing the probability densities illustrates again the importance of the magnetic-field. The breaking of the symmetry about the polarization axis can be seen as the electron density moves in the propagation direction. Figure 4 again shows the probability density after 30 cycles (at the beginning of the turn-off) of the 40-cycle pulse. Along the electric-field polarization axis, the electron can clearly be seen to be localized between $\pm \alpha_{0}= \pm 15$, where $\alpha_{0}=E_{0} / \omega^{2}$ is the classical excursion amplitude of the electron, as would be expected from the HFFT. For the nondipole and counterpropagating cases, the magnetic-field competes with the nuclear force, leading to a series of small escaping wave packets. Note that the force in the propagation direction is zero when the classical displacement along the polarization direction is zero. The influence of the nucleus can also be seen in Fig. 5, where the densities at the end of the 40-cycle pulse are displayed. In the dipole case (upper plot) the wave packet is nearly symmetric about the
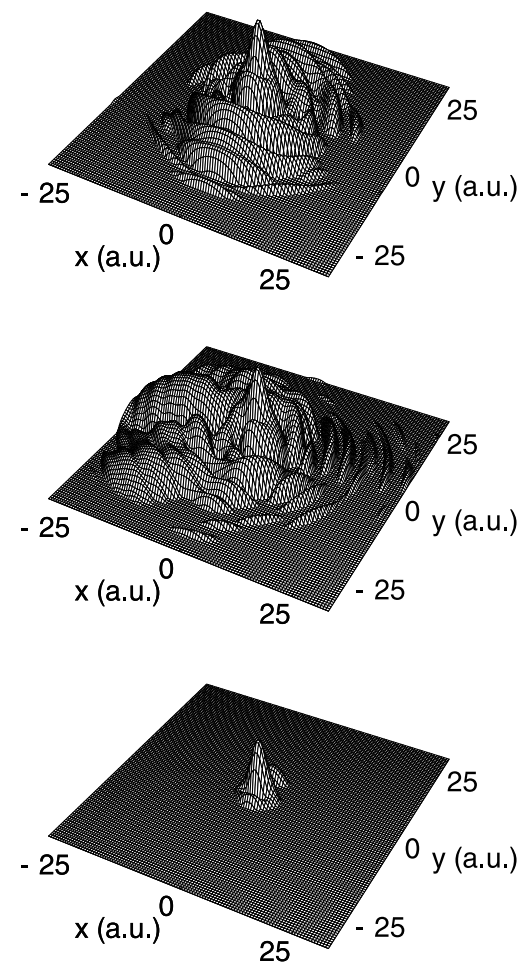

FIG. 5. Same as Fig. 3, except that the probability density is shown at the end of a 40-cycle trapezoidal pulse. In the middle plot, the nucleus is seen to pull the part of the wave function that drifted in the pulse propagation direction back toward the nucleus. However, due to its kinetic energy, this part continues past the nucleus, so that there is a substantial probability of finding the electron at negative values of $x$. For the counterpropagating pulse (lower drawing), little probability density remains in the vicinity of the nucleus.

origin, while in the nondipole case (middle plot) an electron escaping along the propagation axis is now pushed back to the nucleus. However, due to its inertia, it passes to the opposite side of the nucleus.

The survival probability and final-state populations obtained by projecting the wave function at the end of the pulse onto the field-free states are shown in Fig. 6 as functions of the pulse duration. Results are represented by circles (dipole), triangles (nondipole), and squares (counterpropagating). Comparing the dipole and nondipole results, it is evident that the magnetic-field component of the laser pulse strongly influences the stability of the atom, particularly for pulse durations around 16 cycles. For very short pulses, the interaction time is not long enough for the magnetic field to cause a significant displacement of the electron along the propagation axis. However, for the longest pulses considered, the more adiabatic laser-atom interaction means that more population remains in the ground and low excited states of the $\mathrm{KH}$ potential. For the nondipole case, this reduces the effect of the magnetic-field component of the laser. However, for counterpropagating pulses, the survival probability decreases as the laser pulse duration is increased, with two counterpropagating pulses leading to significantly more ionization than a single laser pulse. In the subsequent two sections, we look in more detail at how the pulse duration affects the breakdown of the dipole approximation. 


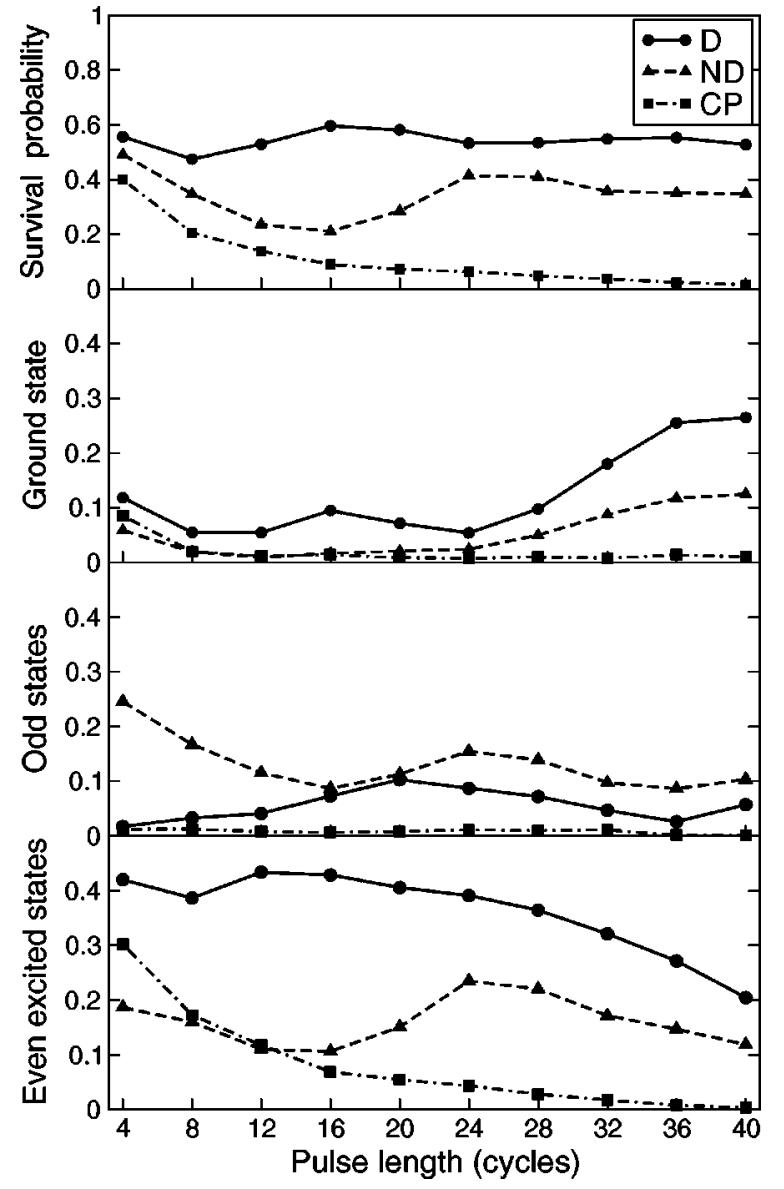

FIG. 6. Survival probability (upper plot), ground-state population (upper middle plot), population in the odd-parity states (lower middle plot), and population in the even-parity excited states (lower plot) at the end of the pulse as functions of the laser pulse duration. As in the previous figures, $E_{0}=15$ and $\omega=1$, and the pulse shape is trapezoidal (see the text). Circles correspond to results obtained in the dipole approximation (D), while triangles and squares are for the nondipole (ND) and counterpropagating (CP) calculations, respectively. Lines between the results are drawn to guide the eye. Note that the difference between the dipole and nondipole survival probabilities is maximum for a pulse length of 16 cycles, with the dipole survival probability being overestimated by more than a factor of 2 .

\section{EFFECT OF THE MAGNETIC-FIELD COMPONENT}

Laser-atom interaction dynamics in the high-frequency, high-intensity regime depend strongly on the duration of the pulse. For pulses that are not ramped on too quickly, the atom can be described by a single Floquet state [29]. Within the framework of the HFFT, the lowest-order approximation of this state would be the ground state of the KH potential [4]. However, when the pulse duration is very short and $E_{0}$ is large, ionization is governed primarily by wave-packet spreading. In this regime, a strong-field approximation can be used to describe the evolution of the system $[11,12,30]$. Between these two regimes, ionization and excitation occur via "shake-up" and "shake-off', processes. Within the HFFT, these processes correspond to nonadiabatic transitions between bound and continuum Floquet eigenstates. In this

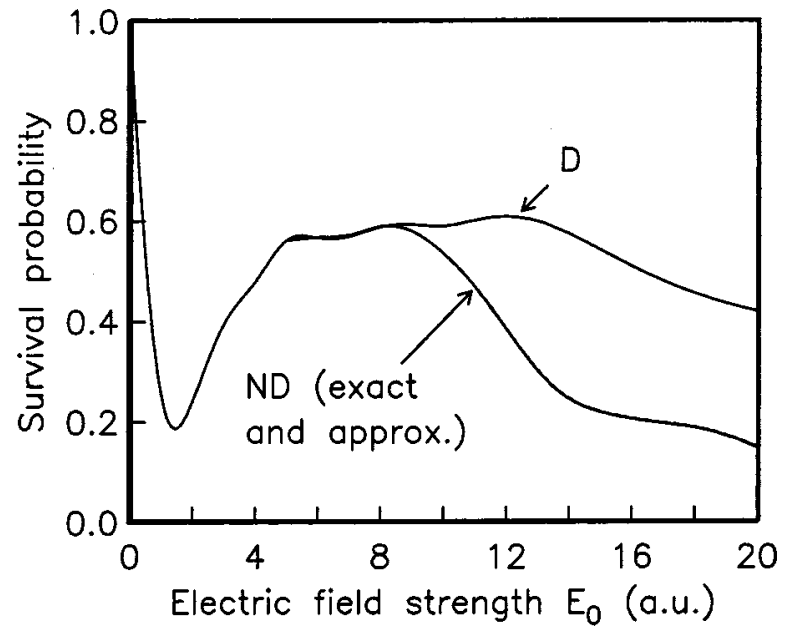

FIG. 7. Survival probability as a function of the maximum electric-field strength, $E_{0}$, of the laser pulse. A 12-cycle trapezoidal pulse (three-cycle turn-on and turn-off, and six-cycle constant intensity) was used, and $\omega=1$. Dipole (D) and nondipole (ND) results are shown, with the nondipole results obtained using the Hamiltonian containing the fully spatially dependent vector potential [see Eq. (5)] and the approximate Hamiltonians given by Eqs. (14) and (15). Differences between the three nondipole calculations cannot be distinguished in the figure.

intermediate regime, the initial evolution of the wave packet is essentially that of a free electron, so long as the width of the wave packet along the polarization direction is much smaller than the classical electron excursion amplitude $[11,12]$.

The range of pulse durations considered here spans the very short pulse regime, where the evolution of the atom is highly nonadiabatic, to the weakly nonadiabatic regime. The origin of the differences between the dipole and nondipole calculations in the very short pulse regime, in particular the breakdown of the dipole approximation at relatively moderate intensities and the improvement of the dipole approximation with increasing frequency, can be understood by considering the motion of a classical electron in the laser field [20]. When the displacement of the wave packet in the propagation direction becomes comparable to the size of the initial state, the dipole approximation is no longer applicable, as discussed above. This is consistent with the probability densities seen in Fig. 1, where the dynamics is essentially that of a spreading free wave packet in the laser pulse.

Figures 2 and 4 illustrate the evolution of the atom in the weakly nonadiabatic regime. To gain a better understanding of the origin of the differences between the dipole and nondipole calculations in this regime, we now investigate the applicability of the long-wavelength approximation introduced above. The approximate Hamiltonians, in particular Eq. (15), have a relatively simple form, and we first assess their accuracy. In Fig. 7 the survival probability is shown as a function of the electric field strength $E_{0}$ for a 12-cycle $(3+6+3$ cycles $)$ pulse. Shown are four curves: the dipole results (D), the nondipole (ND) results using the Hamiltonian containing the exact vector potential, and the Hamiltonians given by Eqs (14) and (15). In the figure, the latter three 
curves cannot be distinguished. At the largest field strength considered, the calculations agree to about $1 \%$ indicating that the long-wavelength approximation, and notably Hamiltonian (15), is an excellent approximation in this intensity and frequency regime. Hence we use Eq. (15) to obtain insight into how the magnetic field influences the stabilization dynamics for longer pulse durations.

We start by introducing the function $\Psi^{\prime}(\mathbf{r}, t)$ by

$$
\Psi(\mathbf{r}, t)=\exp \left[i \frac{x}{2 c} A^{2}(\omega t)\right] \Psi^{\prime}(\mathbf{r}, t) .
$$

Using this expression and Eq. (15), the wave function $\Psi^{\prime}(\mathbf{r}, t)$ is found to be a solution of the time-dependent Schrödinger equation

$$
\begin{aligned}
i \frac{\partial}{\partial t} \Psi^{\prime}(\mathbf{r}, t)= & \left(\frac{1}{2}\left[-i \boldsymbol{\nabla}+\mathbf{A}(\omega t)+\frac{\hat{\mathbf{x}}}{2 c} A^{2}(\omega t)\right]^{2}\right. \\
& +V(r)) \Psi^{\prime}(\mathbf{r}, t) .
\end{aligned}
$$

The spatially homogeneous terms are then transformed away, yielding

$$
\begin{aligned}
i \frac{\partial}{\partial t} \Psi^{\prime \prime}(\mathbf{r}, t)= & \left(-\frac{1}{2} \nabla^{2}-i\left[\mathbf{A}(\omega t)+\frac{\hat{\mathbf{x}}}{2 c} A^{2}(\omega t)\right] \cdot \boldsymbol{\nabla}\right. \\
& +V(r)) \Psi^{\prime \prime}(\mathbf{r}, t),
\end{aligned}
$$

with

$$
\Psi^{\prime \prime}(\mathbf{r}, t)=\exp \left(i \frac{1}{2} \int^{t}\left[\mathbf{A}\left(\omega t^{\prime}\right)+\frac{\hat{\mathbf{x}}}{2 c} A^{2}\left(\omega t^{\prime}\right)\right]^{2} d t^{\prime}\right) \Psi^{\prime}(\mathbf{r}, t) .
$$

Let us now consider this equation during the flat part of the pulse. To lowest order in $1 / c$, the momentum of a classical electron in the laser field is $\mathbf{p}(t)=\mathbf{A}(\omega t)+\hat{\mathbf{x}} A^{2}(\omega t) /(2 c)$ $=\left(2 U_{p} / c \cos ^{2} \omega t, \omega \alpha_{0} \cos \omega t\right)$, where $U_{p}=E_{0}^{2} /\left(4 \omega^{2}\right)$ is the ponderomotive energy, and $\alpha_{0}$ is the classical excursion amplitude. Separating the constant drift in the propagation direction from the oscillating term, we write $\mathbf{p}(t)=\left(U_{p} / c, 0\right)$ $+\left(U_{p} / c \cos 2 \omega t, \omega \alpha_{0} \cos \omega t\right)=\overline{\mathbf{p}}+\mathbf{p}_{0}(t)$, so that

$$
i \frac{\partial}{\partial t} \Psi^{\prime \prime}(\mathbf{r}, t)=\left(-\frac{1}{2} \nabla^{2}-i\left[\overline{\mathbf{p}}+\mathbf{p}_{0}(t)\right] \cdot \boldsymbol{\nabla}+V(r)\right) \Psi^{\prime \prime}(\mathbf{r}, t) .
$$

We next define a $\mathrm{KH}$ frame transformation by $\Psi_{\mathrm{KH}}(\mathbf{r}, t)$ $=\exp [\boldsymbol{\rho}(t) \cdot \boldsymbol{\nabla}] \Psi^{\prime \prime}(\mathbf{r}, t)$, where $\boldsymbol{\rho}(t)=\int_{t_{0}}^{t} \mathbf{p}_{0}\left(t^{\prime}\right) d t^{\prime}$, and $t_{0}$ is the time at the end of the pulse turn-on. Note that $\boldsymbol{\rho}(t)$ describes the figure-of- 8 trajectory of the electron, and does not include the displacement due to the constant drift $\overline{\mathbf{p}}$. In the accelerating $\mathrm{KH}$ frame, Eq. (22) becomes

$$
i \frac{\partial}{\partial t} \Psi_{\mathrm{KH}}(\mathbf{r}, t)=\left(-\frac{1}{2} \nabla^{2}-i \overline{\mathbf{p}} \cdot \boldsymbol{\nabla}+V[|\mathbf{r}-\boldsymbol{\rho}(t)|]\right) \Psi_{\mathrm{KH}}(\mathbf{r}, t) .
$$

Introducing a new wave function $\Psi_{\mathrm{KH}}^{\prime}(\mathbf{r}, t)$, via the gauge transformation

$$
\Psi_{\mathrm{KH}}(\mathbf{r}, t)=\exp \left(-i \overline{\mathbf{p}} \cdot \mathbf{r}+i \frac{1}{2} \bar{p}^{2} t\right) \Psi_{\mathrm{KH}}^{\prime}(\mathbf{r}, t),
$$

yields

$$
i \frac{\partial}{\partial t} \Psi_{\mathrm{KH}}^{\prime}(\mathbf{r}, t)=\left(-\frac{1}{2} \nabla^{2}+V[|\mathbf{r}-\boldsymbol{\rho}(t)|]\right) \Psi_{\mathrm{KH}}^{\prime}(\mathbf{r}, t) .
$$

At this point, a high-frequency approximation can be made, i.e., it is assumed that the dynamics of the system is essentially governed by the cycle average of the potential $V[\mid \mathbf{r}$ $-\boldsymbol{\rho}(t) \mid]$. In the dipole approximation, this entails taking the cycle average of the potential $V[|\mathbf{r}-\boldsymbol{\alpha}(t)|]$, where $\boldsymbol{\alpha}(t)$ $=\left(0, \alpha_{0} \sin \omega t\right)$ describes the simple harmonic motion of the electron along the polarization direction. Since the ratio of the excursion amplitude along the propagation direction to the excursion amplitude along the polarization direction is $E_{0} /(8 c \omega) \simeq 0.01$ for $E_{0}=15$ and $\omega=1$, the eigenstates of the cycle average of the potential $V[|\mathbf{r}-\boldsymbol{\rho}(t)|]$ will differ very little from the eigenstates of the cycle average of the potential $V[|\mathbf{r}-\boldsymbol{\alpha}(t)|][23]$. This also means that the ionization rates of the quasibound states of the two potentials cannot be very different. We therefore replace $V[|\mathbf{r}-\boldsymbol{\rho}(t)|]$ by $V[|\mathbf{r}-\boldsymbol{\alpha}(t)|]$. The high-frequency approximation then gives

$$
i \frac{\partial}{\partial t} \Psi_{\mathrm{KH}}^{\prime}(\mathbf{r}, t) \simeq\left(-\frac{1}{2} \nabla^{2}+V_{0}\left(\mathbf{r}, \alpha_{0}\right)\right) \Psi_{\mathrm{KH}}^{\prime}(\mathbf{r}, t),
$$

where

$$
V_{0}\left(\mathbf{r}, \alpha_{0}\right)=\frac{\omega}{2 \pi} \int_{0}^{2 \pi / \omega} d t V[|\mathbf{r}-\boldsymbol{\alpha}(t)|]
$$

is the static $\mathrm{KH}$ potential. It is seen that $\Psi_{\mathrm{KH}}^{\prime}(\mathbf{r}, t)$ satisfies the time-dependent Schrödinger equation in the dipole and high-frequency approximations. Therefore, for the laser intensities and frequency considered here, the differences in the survival probability between the dipole and nondipole calculations can largely be attributed to nonadiabatic effects during the turn-on and turn-off of the laser pulse. Hence, as the laser turn-on and turn-off become longer, the evolution of the system remains to a larger extent adiabatic and differences between the dipole and nondipole survival probabilities should become smaller. This trend can be seen in the results of Fig. 6. However, it must be borne in mind that the simple model presented here does not appear to be able to explain the series of ionizing wave packets seen in the middle plot in Fig. 4, and that for a 40-cycle pulse the evolution of the atom in the field is not yet adiabatic. The influ- 
ence of the magnetic-field component of the pulse can be reduced further by imposing additional constraints on the form of the laser pulse [27].

\section{EFFECT OF THE MAGNETIC-FIELD COMPONENT: COUNTERPROPAGATING PULSES}

It would appear that the detrimental effect of the magnetic-field could be eliminated by having the atom interact with two counterpropagating laser pulses. Taking an electric field of the form given by Eq. (7), one sees that there are fixed points in space where the magnetic-field vanishes, while the electric field does not. In particular, the electric and magnetic-fields during the flat part of the pulse are

$$
\mathbf{E}\left(\eta, \eta^{\prime}\right)=\frac{E_{0}}{2}\left[\sin \eta+\sin \eta^{\prime}\right] \hat{\mathbf{y}}=E_{0} \cos (k x) \sin (\omega t) \hat{\mathbf{y}}
$$

$$
\mathbf{B}\left(\eta, \eta^{\prime}\right)=\frac{E_{0}}{2 c}\left[\sin \eta-\sin \eta^{\prime}\right] \hat{\mathbf{z}}=-\frac{E_{0}}{c} \sin (k x) \cos (\omega t) \hat{\mathbf{z}}
$$

At $x=0$ the electric field oscillates between $-E_{0}$ and $E_{0}$, but the magnetic-field vanishes for all times. Near $x=0$ the magnetic field, of course, does not vanish. However, we have seen that the magnetic-field component of the laser pulse strongly affects the stabilization dynamics when counterpropagating pulses are used. From our calculations, it is in fact seen that counterpropagating pulses result in survival probabilities that are smaller than those obtained for a single pulse. Classical Monte Carlo simulations show a similar trend [20]. We now look at this in more detail. We first consider the classical dynamics of a free electron in the laser field. This will give insight into the wave-packet dynamics in very short pulses.

The initial ground state of the atom has a small spatial distribution. However, for a pulse with a short turn-on, the wave function evolves essentially as a free wave packet in the laser field. This wave packet spreads considerably within a few laser cycles (see Fig. 1) into regions of space where the magnetic-field can no longer be neglected. To understand its influence on the electron, we consider the Lorentz equation. To lowest order in $x / c$, the force along the $x$ direction on the electron in the laser pulse (and in the absence of the nucleus) is given by

$$
F_{x}(x, t)=\frac{x}{c^{2}} \mathbf{A}(\omega t) \cdot \frac{d}{d t} \mathbf{E}(\omega t) .
$$

The force will always act such that it drives the electron away from the origin along the propagation axis. This is detrimental to stabilization. Note that while the force $F_{x}(x, t)$ is a factor $\sim x \omega / c$ smaller than $F_{x}(t)$ defined in Eq. (9), it is nevertheless comparable to the force due to the $\mathrm{KH}$ potential that binds the electron (in the dipole approximation), as will be discussed below. Let us consider an electron initially at rest at the magnetic-field node $x=0$. According to the Lorentz force, the velocity along the $x$ axis will always be $v_{x}$

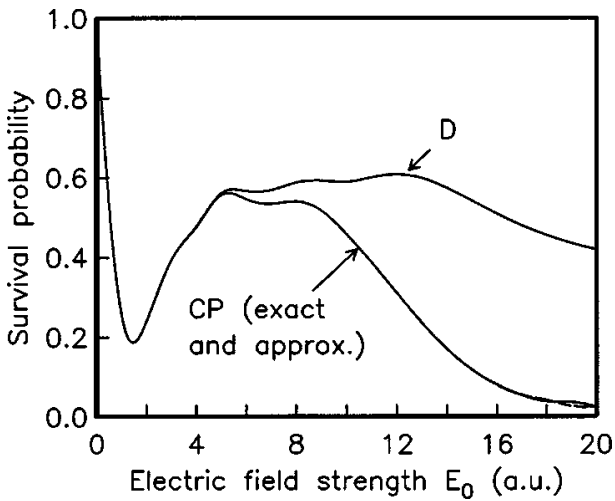

FIG. 8. Same as Fig. 7, but survival probabilities in the dipole approximation (D) are now compared with results for two counterpropagating $(\mathrm{CP})$ laser pulses. The latter results were obtained using the Hamiltonian with the fully spatially dependent vector potential, as well as the approximate Hamiltonians given by Eqs (16) and (17). For $E_{0}<17$, differences between these three calculations cannot be distinguished in the figure.

$=0$. However, these trajectories are not stable. Introducing a small displacement $\delta x$ such that $k \delta x \ll 1$ and the perturbation $\delta v_{x}$ in the velocity along the $x$ direction, the linearized equation of motion reads

$$
\frac{d \delta v_{x}}{d t} \simeq-\frac{E_{0}^{2} \delta x}{c^{2}} \cos ^{2}(\omega t)
$$

This equation can be solved in terms of even and odd Mathieu functions. The solutions are always exponentially growing, and the system is therefore unstable. We note that this linear stability analysis is one way to show (close to $x$ $=0$ ) the well-known fact that the ponderomotive force tends to concentrate electrons in regions where the electric field is smallest.

We now look at the effect of counterpropagating pulses from a quantum-mechanical point of view. As for the case of a single pulse, we wish to gain insight into the weakly nonadiabatic regime. Again, we first assess the applicability of the long-wavelength approximations for the Hamiltonian, Eqs (16) and (17). As in Fig. 7, four curves are plotted in Fig. 8. However, now the dipole results (D) are compared to the counterpropagating $(\mathrm{CP})$ results using a Hamiltonian containing the exact vector potential and the approximate Hamiltonians given by Eqs (16) and (17). Overall, the agreement between the three sets of results is excellent, with only slight differences appearing between the exact calculation and the two approximate calculations when $E_{0}>17$. Hence we proceed by using the Hamiltonian given by Eq. (17). In the time-dependent Schrödinger equation, as in Sec. VI we transform away the spatially homogeneous term in the Hamiltonian. Defining the KH transformation by $\Psi^{\mathrm{KH}}(\mathbf{r}, t)$ $=\exp [\boldsymbol{\alpha}(t) \cdot \boldsymbol{\nabla}] \Psi(\mathbf{r}, t)$, we now have

$$
\begin{aligned}
i \frac{\partial}{\partial t} \Psi_{\mathrm{KH}}(\mathbf{r}, t)= & \left(-\frac{1}{2} \nabla^{2}-\frac{1}{2} \frac{x^{2}}{c^{2}} \mathbf{A}(\omega t) \cdot \frac{d}{d t} \mathbf{E}(\omega t)\right. \\
& +V[|\mathbf{r}-\boldsymbol{\alpha}(t)|]) \Psi_{\mathrm{KH}}(\mathbf{r}, t) .
\end{aligned}
$$




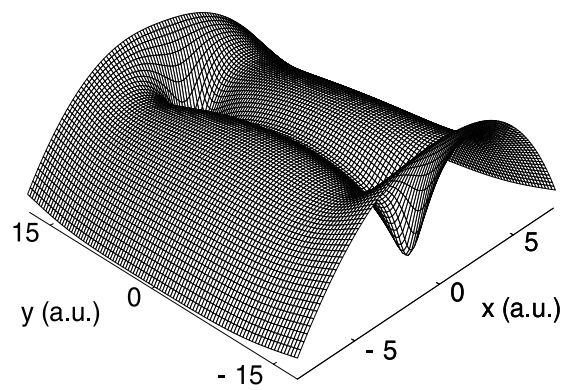

FIG. 9. Surface plot of the effective potential given by Eq. (34), with $E_{0}=\alpha_{0}=15$.

We next consider the wave function during the flat part of the pulse. Making the high-frequency approximation yields

$$
\begin{aligned}
i \frac{\partial}{\partial t} \Psi_{\mathrm{KH}}(\mathbf{r}, t) \simeq & \left(-\frac{1}{2} \nabla^{2}-\frac{E_{0}^{2} x^{2}}{4 c^{2}}[1+\cos 2 \omega t]\right. \\
& \left.+V_{0}\left(\mathbf{r}, \alpha_{0}\right)\right) \Psi_{\mathrm{KH}}(\mathbf{r}, t) .
\end{aligned}
$$

Ignoring the rapidly oscillating term, we see that the system is governed by the effective time-independent potential

$$
V_{\mathrm{eff}}(\mathbf{r})=-E_{0}^{2} x^{2} /\left(4 c^{2}\right)+V_{0}\left(\mathbf{r}, \alpha_{0}\right)
$$

The sum of the two potentials gives rise to a potential barrier in the propagation direction, so that the bound states of the potential $V_{0}\left(\mathbf{r}, \alpha_{0}\right)$ can ionize by tunneling. The effective potential $V_{\text {eff }}(\mathbf{r})$ is plotted in Fig. 9 for $E_{0}=\alpha_{0}=15$.

In Fig. 6, it is seen that increasing the laser pulse duration results, for the dipole and nondipole calculations, in more population in the ground state and comparatively less population in excited states at the end of the pulse. The increasingly adiabatic laser-atom interaction increases the probability that the wave function will evolve into the ground state of the $\mathrm{KH}$ potential, and decreases the probability that "shakeoff' ionization will occur. The net result is that the probability of remaining in the ground state at the end of the pulse is larger, in spite of the fact that the atom interacts with the laser pulse for a longer time. This is not the case for the counterpropagating pulses. The results in Fig. 6 show that making the laser-atom interaction longer does not increase the probability of finding the atom in the ground state after the pulse. In this case, the greater probability of remaining in the ground state of the $\mathrm{KH}$ potential due to the more adiabatic pulse turn-on is negated by the relatively large tunneling rate of the wave function through the potential barrier. We also note that the additional nondipole term appearing in the Hamiltonian is proportional to the intensity of the laser field, while the "average depth' of the cycle-averaged $\mathrm{KH}$ potential $V_{0}\left(\mathbf{r}, \alpha_{0}\right)$ decreases as $\alpha_{0}$ increases. This means that even within an adiabatic picture, the system quickly becomes increasingly unstable as the laser intensity increases, since the potential barrier formed by the sum of the two potentials is lowered.
Finally, considering the fact that for counterpropagating pulses a correction of order $1 / c^{2}$ has a larger influence than the correction of order $1 / c$ for a single pulse, one may wonder why the correction of order $1 / c^{2}$ may be neglected in the latter case. Using the long-wavelength expansion [Eq. (12)], the Hamiltonian for a single traveling pulse is, to order $1 / c^{2}$,

$$
H \simeq \frac{1}{2}[\mathbf{p}+\mathbf{A}(\omega t)]^{2}-\left[\frac{x}{2 c} \frac{d}{d t}-\frac{x^{2}}{4 c^{2}} \frac{d^{2}}{d t^{2}}\right] A^{2}(\omega t)+V(r) .
$$

During the flat part of the pulse, $d^{2} A^{2}(\omega t) / d t^{2}=$ $-2 E_{0}^{2} \cos 2 \omega t$. Classically, it is easily shown that the term in $1 / c^{2}$ gives rise to a small correction that, compared to the correction in $1 / c$ can be neglected, in accordance with the results of the quantum mechanical calculations shown in Fig. 7.

\section{CONCLUSIONS}

We have presented results of numerical simulations performed with a two-dimensional model atom interacting with an intense laser pulse in which the magnetic-field component of the laser pulse is included (i.e., the electric-dipole approximation is not assumed). We have demonstrated that the magnetic-field induces a drift along the propagation direction that is sufficient to disrupt the electron dynamics required for atomic stabilization. The influence of the laser pulse duration on the breakdown of the dipole approximation was analyzed by comparing with calculations in the dipole approximation. Significant differences were found, particularly for pulse durations of around 16 cycles. We have also introduced and tested a simple long-wavelength approximation. The accuracy of this approximation makes it a natural starting point for extending existing stabilization models so that the effect of the magnetic-field component of the laser pulse is accounted for. We have shown that having the atom interact with a standing wave, with the atom placed in a node of the magnetic field, leads to even more ionization than the case in which an atom interacts with a single laser pulse of the same intensity. This surprising result is explained in the very short pulse regime by the fact that a classical electron's dynamics is unstable at the node, while for pulses with a longer turn-on it is due to the quantum tunneling through an effective potential barrier determined by a cycle-averaged potential in the $\mathrm{KH}$ frame.

\section{ACKNOWLEDGMENTS}

This work was supported by the UK Engineering and Physical Sciences Research Council (EPSRC) and the Spanish Dirección General de Enseñanza Superior e Investigación Científica (Grant No. PB98-0268). Two of us (J. R. V. D. A. and L. R.) thank W. Becker, J. H. Eberly, L. Plaja, J. San Román, H. R. Reiss, and L. Santos for fruitful discussions, while one of us (N. J. K.) benefited from discussions with $\mathrm{H}$. G. Muller. R. M. Potvliege is thanked for his comments on the manuscript. 
[1] For reviews, see M. Protopapas, C.H. Keitel, and P.L. Knight, Rep. Prog. Phys. 60, 389 (1997); C.J. Joachain, M. Dörr, and N.J. Kylstra, Adv. At., Mol., Opt. Phys. 42, 225 (2000).

[2] J.J. Gersten and M.H. Mittleman, J. Phys. B 9, 2561 (1976).

[3] M. Gavrila and J. Kaminski, Phys. Rev. Lett. 52, 613 (1984); M. Gavrila, M.J. Offerhaus, and J. Kaminski, Phys. Lett. A 118, 331 (1986).

[4] M. Pont, N.R. Walet, M. Gavrila, and C.W. McCurdy, Phys. Rev. Lett. 61, 939 (1988); M. Pont, N.R. Walet, and M. Gavrila, Phys. Rev. A 41, 477 (1990); M. Pont and M. Gavrila, Phys. Rev. Lett. 65, 2362 (1990); M. Gavrila, in Atoms in Intense Laser Fields, edited by M. Gavrila (Academic Press, San Diego, 1992), p. 435; in Multiphoton Processes, edited by L.F. DiMauro, R.R. Freemen, and K. Kulander, AIP Conf. Proc. No. 525 (AIP, Melville, NY, 2000), p. 103.

[5] M. Dörr, R.M. Potvliege, D. Proulx, and R. Shakeshaft, Phys. Rev. A 43, 3729 (1991).

[6] Q. Su, J.H. Eberly, and J. Javanainen, Phys. Rev. Lett. 64, 862 (1991); Q. Su and J.H. Eberly, Phys. Rev. A 43, 2474 (1991); C.K. Law, Q. Su, and J.H. Eberly, ibid. 44, 7844 (1991); Q. Su, A. Sanpera, and L. Roso-Franco, Int. J. Mod. Phys. B 8, 1655 (1994); Q. Su, B.P. Irving, C.W. Johnson, and J.H. Eberly, J. Phys. B 29, 5755 (1996).

[7] A. Patel, M. Protopapas, D.G. Lappas, and P.L. Knight, Phys. Rev. A 58, R2652 (1998).

[8] K.C. Kulander, K.J. Schafer, and J.L. Krause, Phys. Rev. Lett. 66, 2601 (1991).

[9] J.H. Eberly and K.C. Kulander, Science 262, 1229 (1993).

[10] V.C. Reed, P.L. Knight, and K. Burnett, Phys. Rev. Lett. 67, 1415 (1991); R.M.A. Vivirito and P.L. Knight, J. Phys. B 28, 4357 (1995).

[11] R. Grobe and M. Fedorov, Phys. Rev. Lett. 68, 2592 (1992); 69, 3591(E) (1992).

[12] A.S. Patel, N.J. Kylstra, and P.L. Knight, J. Phys. B 32, 5759 (1999).

[13] M.P. de Boer, J.H. Hoogenraad, R.B. Vrijen, L.D. Noordam, and H.G. Muller, Phys. Rev. Lett. 71, 3263 (1993); M.P. Boer, J.H. Hoogenraad, R.B. Vrijen, R.C. Constantinescu, L.D. Noordam, and H.G. Muller, Phys. Rev. A 50, 4085 (1994); N.J. Druten, R.C. Constantinescu, J.M. Schins, H. Nieuwen- huize, and H.G. Muller, ibid. 55, 622 (1997); also see B. Piraux and R.M. Potvliege, ibid. 57, 5009 (1998).

[14] J. Grochmalicki, M. Lewenstein, and K. Rza̧żewski, Phys. Rev. Lett. 66, 1038 (1991); T. Katsouleas and W.B. Mori, ibid. 70, 1561 (1993).

[15] A. Bugacov, M. Pont, and R. Shakeshaft, Phys. Rev. A 48, R4027 (1993); O. Latinne, C.J. Joachain, and M. Dörr, Europhys. Lett. 26, 333 (1994); R.M. Potvliege, Laser Phys. 10, 143 (2000).

[16] C.H. Keitel and P.L. Knight, Phys. Rev. A 51, 1420 (1995).

[17] U.W. Rathe, C.H. Keitel, M. Protopapas, and P.L. Knight, J. Phys. B 30, L531 (1997).

[18] J.R. Vázquez de Aldana and L. Roso, Opt. Express 5, 144 (1999); Phys. Rev. A 61, 043403 (2000).

[19] A.V. Kim, M. Yu. Ryabikin, and A.M. Sergeev, Usp. Fiz. Nauk. 169, 58 (1999) [Phys. Usp. 42, 54 (1999)].

[20] N.J. Kylstra, R.A. Worthington, A. Patel, P.L. Knight, J.R. Vázquez de Aldana, and L. Roso, Phys. Rev. Lett. 85, 1835 (2000).

[21] M. Yu. Ryabikin and A.M. Sergeev, Opt. Express 7, 417 (2000).

[22] The influence of the magnetic-field component of the laser pulse on harmonic generation was recently investigated to order 1/c. See M.W. Walser, C.H. Keitel, A. Scrinzi, and T. Brabec, Phys. Rev. Lett. 85, 5082 (2000); N.J. Kylstra, R.M. Potvliege, and C.J. Joachain, J. Phys. B 34, L55 (2001).

[23] P.S. Krstić and M.H. Mittleman, Phys. Rev. A 42, 4037 (1990).

[24] J.R. Vázquez de Aldana and L. Roso, J. Phys. B 33, 3701 (2000).

[25] W.R. Johnson, S.A. Blundell, and J. Sapirstein, Phys. Rev. A 37, 307 (1988); C.J. Joachain and N.J. Kylstra, J. Phys. B 28, L477 (1995).

[26] J. Bergou and S. Varro, J. Phys. A 13, 2823 (1980); K. Druhl and J.K. McIver, J. Math. Phys. 24, 705 (1983).

[27] H. G. Muller (private communication).

[28] H.R. Reiss, Phys. Rev. A 63, 013409 (2000).

[29] M. Dörr and R.M. Potvliege, J. Phys. B 33, L233 (2000).

[30] M. Dörr, N.J. Kylstra, and R.M. Potvliege, Laser Phys. 11, 250 (2001). 